\title{
Development of an IgG/IgY sandwich-ELISA for the bioactive polypeptide Prothymosin alpha
}

\author{
Chrysoula-Evangelia Karachaliou ${ }^{1}$, Persefoni Klimetzou ${ }^{1}$, Maria Paravatou-Petsotas ${ }^{1}$, Ourania Tsitsilonis ${ }^{2}$, Wolfgang \\ Voelter $^{3}$, Hubert Kalbacher ${ }^{3}$, Christos Zikos ${ }^{1}$, Evangelia Livaniou ${ }^{1}$ \\ ${ }^{1}$ National Centre for Scientific Research ”Demokritos”, INRASTES, Greece \\ ${ }^{2}$ Departmentof Biology, University of Athens, Greece \\ ${ }^{3}$ Interfaculty Institute of Biochemistry, University of Tuebingen, Germany
}

https://doi.org/10.17952/35EPS.2018.230

\section{Introduction}

Prothymosin alpha (ProT $\alpha$ ) is a highly acidic polypeptide expressed in all mammalian cells and consisting of 109 amino acids in humans. The polypeptide exerts important biological functions acting both intracellularly, as a chromatin-remodeling, proliferation-enhancing and anti-apoptotic factor, and extracellularly, as a pleiotropically-operating immunostimulating mediator [1]. Under certain cellular conditions ProT $\alpha$ may be enzymatically cleaved resulting in biologically active peptidyl fragments, such as the N-terminal region $\operatorname{ProT} \alpha$ [1-28] (also known as thymosin alpha 1, T $\alpha 1$ ) and the C-terminal decapeptide ProT $\alpha$ [100-109]. More specifically, it has been reported that the ProT $\alpha[1-28]$ fragment may be formed after proteolytic cleavage of ProT $\alpha$ by a lysosomal asparaginyl endopeptidase [2], while ProT $\alpha$ [100-109] may be produced after ProT $\alpha$ 's cleavage by caspace-3 [3]. Therefore, immunoassays distinguishing between intact parental ProT $\alpha$ and the above mentioned active fragments thereof in specific biological samples are considered valuable laboratory tools, especially for elucidating the precise mechanism(s) of action of the polypeptide.

\section{Experimental/Results}

In the current work we have employed rabbit antibodies G (IgGs) and chicken antibodies Y (IgYs), which were previously raised by our teams, to develop a prototype sandwich-ELISA (Figure 1-A). This assay has proved to be highly specific for the intact ProT $\alpha$ molecule. Briefly, the assay steps are as follows: IgG-antibodies raised in rabbits against a conjugate of synthetic ProT $\alpha$ [100-109] with keyhole limpet hemocyanin (KLH) have been indirectly immobilized on the ELISA microwells. After blocking the remaining binding sites, ProT $\alpha$ solutions of known concentration $(1 \mu \mathrm{g} / \mathrm{mL}-1 \mathrm{ng} / \mathrm{mL})$, prepared by dissolving commercially available ProT $\alpha$ in various cell culture media, were transferred to the plate for incubation. IgY-antibodies raised in chickens against a ProT $\alpha / \mathrm{KLH}$ conjugate which show high specificity for the intact polypeptide $[4,5]$ have been subsequently incubated with the microwells. Finally, commercially available enzyme-labelled secondary anti-IgY antibodies along with the proper chromogenic substrate have been used for signal development. The ProT $\alpha$ sandwichELISA developed shows high reproducibility (inter-assay $\mathrm{CV}<5 \%$ ) and a detection limit of $<2 \mathrm{ng} / \mathrm{mL}$ (Figure $1-B)$. Moreover, assay specificity for the intact $\operatorname{ProT} \alpha$ molecule is very high as verified by cross-reactivity studies with synthetic ProT $\alpha$ [100-109] (Figure 1-B) and ProT $\alpha$ [1-28] as well as synthetic peptides of the beta-thymosin family (data not shown here). 

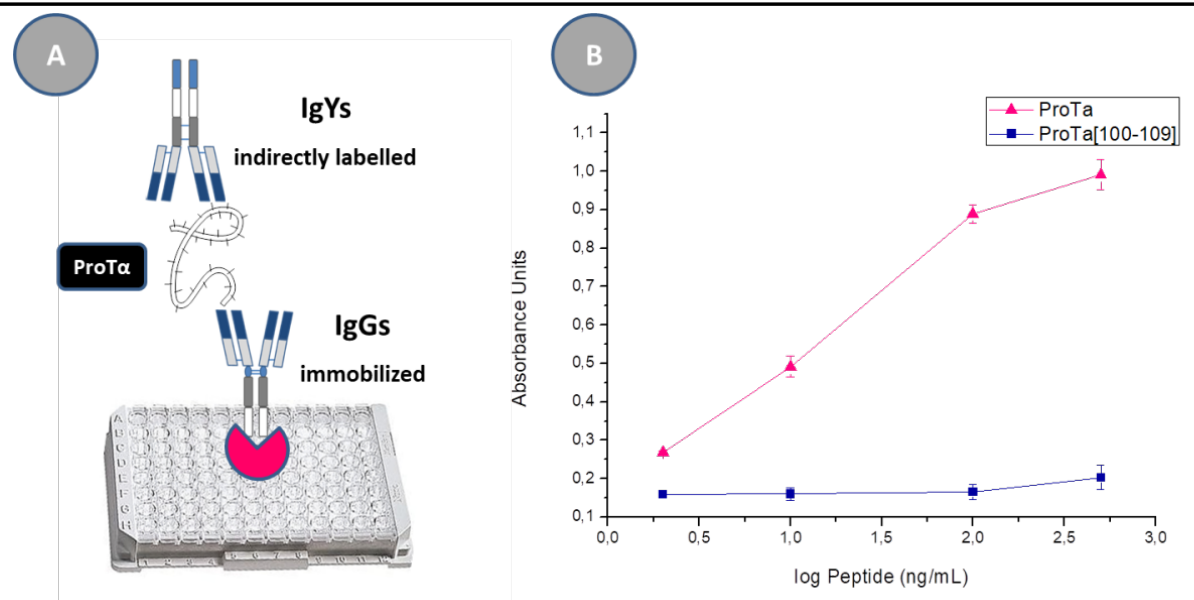

Figure 1: A. Schematic representation of the IgG/IgY-based sandwich-ELISA setup for prothymosin alpha. $B$. Standard curve of ProT $\alpha$ (pink triangle) in the sandwich-ELISA setup over a concentration range of $1 \mathrm{ng} / \mathrm{mL}$ $500 \mathrm{ng} / \mathrm{mL}$. The corresponding curve of synthetic ProTa [100-109] (blue square) verifies no cross-reactivity of this fragment with the ELISA system.

\section{Discussion/Future perspectives}

ProT $\alpha$ is a highly conserved among mammalian organisms polypeptide that in humans may serve as a specific biomarker and/or a target for developing new therapies, especially in cancer and immune diseases. In this work, we have exploited antibodies $G$ and antibodies Y previously raised by our groups against ProT $\alpha$ [100-109]/KLH and ProT $\alpha / \mathrm{KLH}$, respectively, to develop a prototype sandwich-ELISA with high specificity for intact ProT $\alpha$, which exhibits practically no cross-reaction with bioactive fragments of the parental molecule. Moreover, the assay is sensitive and highly reproducible. If necessary, assay sensitivity may be further increased, e.g. using different enzyme substrate systems such as chemiluminescence-based systems. The developed sandwich-ELISA is currently being applied to specific biological samples, i.e. the supernatants of human cells and cells of cancer cell lines, cultured in the presence of various apoptotic/necroticfactors, in order to verify the literature scenario, according to which human cells may release intact $\operatorname{ProT} \alpha$ as a damage-associated molecular pattern (DAMP)/alarmin, under certain survival-threatening circumstances [1]. Potentially, other biological samples, e.g. human sera, may be also analyzed, after slightly modifying the assay procedure. The results obtained after analyzing suitably selected biological samples through the above sandwich ELISA will contribute to the elucidation of the mechanism(s) of action of ProT $\alpha$ under certain conditions, which is a prerequisite for any further clinical exploitation of the polypeptide.

\section{Acknowledgements}

This research is implemented through IKY scholarships program and co-financed by the European Union (European Social Fund - ESF) and Greek national funds through the action entitled "Reinforcement of Postdoc-toral Researchers", in the framework of the Operational Program "Human Resources Development Program, Education and Lifelong Learning" of the National Strategic Reference Framework (NSRF) 2014 2020. We also acknowledge partial support of this work by the project "NCSRD - INRASTES research activities in the framework of the national RIS3" (MIS 5002559) which is implemented under the "Action for the Strategic Development on the Research and Technological Sector", funded by the Operational Program "Competitiveness, Entrepreneurship and Innovation" (NSRF 2014-2020) and co-financed by Greece and the European Union (European Regional Development Fund).

\section{References}

[1] Samara P, Karachaliou CE, Ioannou K et al., Prothymosin alpha: An alarmin and more..., Current Medicinal Chemistry. 2017;24(17):1747-60; doi: 10.2174/0929867324666170518110033

[2] Sarandeses CS et al., Prothymosin alpha is processed to thymosin alpha 1 and thymosin alpha 11 by a 
lysosomal asparaginyl endopeptidase, Journal of Biological Chemistry. 2003 Apr 11;278(15):13286-93; doi: 10.1074/jbc.M213005200

[3] Evstafieva AG et al., Prothymosin alpha fragmentation in apoptosis, FEBS Letters. 2000 Feb 11;467(23):150-4; doi.org/10.1016/S0014-5793(00)01139-X

[4] Klimentzou P. et al., Development and immunochemical evaluation of antibodies $Y$ for the poorly immunogenic polypeptide prothymosin alpha, Peptides. 2006 Jan;27(1):183-93; doi: 10.1016/ j.peptides.2005.07.002

[5] Karachaliou CE et al., Highly specific polyclonal antibodies $Y$ for intact prothymosin alpha, a humanpolypeptide with diagnostic and/ortherapeutic potential, Journal of Biotechnology. 2018; 280S:S70; doi.org/10.1016/ j.jbiotec.2018.06.228 